\title{
A produção de vacinas é estratégica para o Brasil
}

\section{The production of vaccines is a strategic activity for Brazil}

\author{
Organização Pan-Americana da Saúde \\ Setor de Embaixadas Norte, Lote 19 \\ 70800-400 Brasília - DF Brasil \\ risi@bra.ops-oms.org
}

Entrevista com/Interview with João Baptista Risi Júnior

RISI Jr., J. B.: 'A produção de vacinas é estratégica para o Brasil', entrevista concedida a Carlos Fidelis Ponte. História, Ciências, Saúde - Manguinhos, vol. 10 (suplemento 2): 771-83, 2003.

A produção de imunobiológicos deveria ser assumida pelo governo federal como atividade estratégica para o país e tratada em plano diretor que instituísse mecanismos para assegurar competitividade aos laboratórios nacionais. É o que sustenta, nesta entrevista, o médico João Baptista Risi Júnior, uma das maiores autoridades em epidemiologia do Brasil. Fluminense de Niterói, Risi Júnior é um vasto arquivo humano da história da imunização brasileira. Com as credenciais de quem coordenou a Campanha de Erradicação da Varíola no estado do Rio de Janeiro (de 1968 a 1970), chefiou o Setor de Epidemiologia da Fundação SESP (de 1974 a 1979), foi secretário nacional de Ações Básicas de Saúde (de 1980 a 1988) e por duas vezes secretário nacional de Vigilância Sanitária (em 1992 e 1999), ele se recusa a situar em campos opostos a estratégia dos dias nacionais de vacinação e a defesa dos serviços permanentes de atenção integral, universal e descentralizada de saúde.

O atual representante no Brasil da Organização Pan-Americana de Saúde (OPAS) preconiza o uso de todos os recursos possíveis e a convivência da gestão centralizada, necessária à padronização do Brasil continental, com a ação local, descentralizada, prioridade do Sistema ùnico de Saúde (SUS). Neste depoimento concedido a Carlos Fidélis Ponte, ele também não poupou críticas à instabilidade institucional e à descontinuidade administrativa, pelo desperdício de experiências históricas que, consideradas no presente, ajudariam a reduzir os erros e a multiplicar os acertos no serviço público de atendimento integral à saúde. Erros e acertos que, no encontro de contas, põem em jogo vidas humanas.

PALAVRAS-CHAVE: vacina, varíola, poliomielite, CEME, Fundação SESP, prevenção, imunização, campanha, saúde.

RISI Jr., J. B.: 'The production of vaccines is a strategic activity for Brazil'. Interview conducted by Carlos Fidelis Ponte. História, Ciências, Saúde - Manguinhos, vol. 10 (supplement 2): 771-83, 2003.

The production of immunobiological vaccines should be recognized by the federal government as a strategic activity for the country and should be ruled by a national planning program that would create the means to guarantee competitive conditions to the national pharmaceutical industry. These are the points stressed out during this interview with medical doctor João Baptista Risi Jünior, one of the highest authorities in epidemiology in Brazil.

Born in Niterói, Risi Junior is a vast human archive on the history of immunization in the country. From 1968 to 1970, he coordinated the Campanha de Erradicação da Variola (Smallpox Erradication Campaign) in Rio de Janeiro State. From 1974 to 1979, he was the head of SESP Foundation Epidemiology Department. From 1980 to 1988, Risi Junior was the national secretary of Ação Básica de Saúde (Basic Health Actions) and twice (1992 and 1999), Vigilância Sanitária (Sanitary Watch) national secretary. As the authority he unquestionably is, Risi Junior refuses to place 'natinal vaccination day' strategy apart from the claim for permanent, integral, universal and decentralized health services.

Risi, the current representative of Brazil in the Pan-American Health Organization, strongly recommends the investment of all available resources and the interaction between a centralized administration, which is necessary for the development of standards throughout continental Brazil, and decentralized local actions, which is part of SUS priorities. Interviewed by Carlos Fidelis Ponte, he greatly criticized institutional instability and administrative discontinuity, which according to him are due to the disregarding of historical experiences. If they were taken into account, those experiences would help to diminish the mistakes and multiply the good results of public health services. After all, these mistakes and good results make a difference to buman lives.

KEYWORDS: vaccine, smallpox, polyomyelitis, CEME, Fundação SESP, prevention, immunization, campaign, health. 
Qual foi a grande inovação que o Programa Nacional de Imunização (PNI) trouxe para as açôes de controle das doenças imunopreveniveis?

A criação do PNI, em 1973, representou o desfecho de uma série de iniciativas do Ministério da Saúde na área do controle de doenças. Ela foi fortemente influenciada pela criação da Central de Medicamentos (CEME), dois anos antes, em 71, encarregada de implantar a política de assistência farmacêutica no país. Até então, a administração de vacinas era realizada de diferentes modos, com maior ou menor participação do governo federal. Isto porque trinta anos antes, em 1941, a reforma administrativa consolidara a política federal no controle de algumas doenças transmissíveis e a vacinação contra essas doenças passou a ser gerenciada por órgãos autônomos, que assumiam a responsabilidade pela produção, normalização técnica e utilização dos produtos. As vacinas BCG e contra a febre amarela, por exemplo, estavam a cargo, respectivamente, dos serviços nacionais de tuberculose e de febre amarela. Outras vacinas, não vinculadas aos serviços nacionais, estavam sob a responsabilidade da rede de centros de saúde, em geral administrada pelos governos estaduais, embora também existissem redes autônomas, como a da Fundação SESP, presente em todos os estados. O governo federal cooperava tecnicamente e apoiava o suprimento de imunizantes, mas de forma insuficiente e descontínua. Qualquer esforço para ampliar coberturas vacinais dependia da iniciativa própria dos governos estaduais. A ação federal era episódica, movida por compromissos internacionais, como o controle da varíola ou o surgimento de novas vacinas, como as da poliomielite e do sarampo, já na década de 1960.

\section{Que elementos concorreram para a criação do PNI?}

Duas iniciativas nacionais foram particularmente importantes: a Campanha de Erradicação da Varíola (CEV) e o Plano Nacional de Controle da Poliomielite (PNCP). Ambas tinham como estratégia básica a vacinação extensiva da população e exigiam estreita colaboração da rede de serviços de saúde, onde essas ações eram rotineiramente executadas. Ambas também tiveram em comum o fato de serem organizadas e dirigidas por técnicos da Fundação SESP, instituição notabilizada na administração de serviços integrais de saúde no Brasil. No caso da varíola, a aproximação deu-se, inicialmente, para organizar um sistema de vigilância epidemiológica da doença, a partir de 1969. Mas conforme chegava o fim da campanha, houve a preocupação de manter a vacinação de rotina, para assegurar que as coberturas permanecessem elevadas. Já o Plano Nacional de Controle da Poliomielite foi concebido em 1971 e muito influenciado pela experiência da CEV, tendo buscado aperfeiçoar a estratégia de vacinação em massa, com esforços integrados de mobilização institucional e social nos estados. Então a SESP também teve 
"Na década de 1960, o Brasil era o único país endêmico no continente americano. A primeira tentativa nacional de controlar a varíola, em 1962, fracassou por falta de infraestrutura capaz de atuar em todo o território nacional." participação fundamental na criação de uma importante base de apoio a essa nova estratégia de atuação, que a partir de 1970 serviu de modelo nacional: a unidade de vigilância epidemiológica da Secretaria de Saúde do Rio Grande do Sul.

\section{O que levou o Ministério da Saúde a defender a criação do PNI?}

No quadro institucional do Ministério da Saúde, no início da década de 1970, havia clara dificuldade de unificar as ações de imunização em um programa integrado. As duas principais experiências mencionadas — a CEV e o PNCP — estavam sediadas em órgãos recém-criados na reforma administrativa de 1970, e que mesclavam culturas institucionais muito distintas. Um deles era a Superintendência de Campanhas de Saúde Pública (Sucam), que absorvera a CEV junto com o Departamento Nacional de Endemias Rurais (DNERU) e a Campanha de Erradicação da Malária (CEM). O outro era o Departamento Nacional de Profilaxia e Controle de Doenças (DNPCD), que concebeu e implementou o Plano Nacional de Controle da Poliomielite, como responsabilidade da nova Divisão Nacional de Epidemiologia e Estatística de Saúde (DNEES).

\section{Qual o papel da CEME na implantação do PNI?}

A convergência de esforços na área de imunização foi induzida pela Central de Medicamentos (CEME), que, vinculada à Presidência da República, tinha por função estratégica organizar a produção, o controle de qualidade e o suprimento de medicamentos essenciais, inclusive produtos imunobiológicos. O planejamento da ação da CEME requeria, naturalmente, a previsão integrada dos quantitativos de produtos necessários, forçando o Ministério da Saúde a se articular internamente para tal fim. A oportunidade de criar o PNI surgiu com a administração que assumiu o Ministério da Saúde em 1972 (gestão de Mário Machado de Lemos), voltada para a formulação de grandes programas estratégicos. O órgão incumbido de elaborar o PNI foi o Departamento Nacional de Profilaxia e Controle de Doenças, por intermédio da Divisão Nacional de Epidemiologia e Estatística de Saúde, com apoio de técnicos da CEME.

As campanhas de combate à febre amarela, à malária e à varíola influenciaram a metodologia e o desenvolvimento do PNI?

Os chamados "programas verticais", tão criticados a partir da década de 1970, foram na realidade os que deram dimensão nacional à saúde pública brasileira, que até então atuava no espaço geográfico do Distrito Federal, de São Paulo e de alguns outros municípios. Esses programas traçaram objetivos nacionais e propiciaram $\mathrm{O}$ desenvolvimento de modelos capazes de superar imensas barreiras geográficas, político-administrativas e culturais, que impediam o uso eficaz das tecnologias disponíveis, que exigiam procedimentos 
“A partir de

1974, quando o

Ministério da

Saúde foi para

Brasília, houve

retrocesso na

cobertura do

PNI. Pretendeu-

se fortalecer a

vacinação de

rotina, em

substituição às

campanhas. Essa

política não se

mostrou efetiva,

pois a rede de

serviços estava

despreparada. As

coberturas

permaneceram

insatisfatórias, o

que propiciou

várias epidemias, entre elas a da poliomielite." padronizados. A experiência acumulada nesse processo foi mais expressiva no combate aos vetores da febre amarela e da malária, desde o início do século XX, e teve a cooperação internacional, em especial da Fundação Rockefeller.

Por que na área da imunização os avanços foram mais lentos?

Porque nesta área os avanços dependiam da atuação de serviços permanentes de saúde, que eram insuficientemente distribuídos e pouco resolutivos. O caso da varíola é emblemático. Na década de 1960, o Brasil era o único país endêmico no continente americano. A primeira tentativa nacional de controlar a doença, em 1962, baseou-se na mobilização de recursos locais e comunitários, com pouco investimento federal, e fracassou por falta de infra-estrutura capaz de atuar em todo o território nacional e de manter esforços continuados.

\section{A que o senhor atribui o notório êxito da CEV, realizada em 1966?}

A CEV foi instituída sob forte pressão internacional e introduziu métodos que foram bem-sucedidos e que se baseavam em sólida estrutura administrativa e na organização do trabalho de campo, com visitação domiciliar. É interessante notar que a direção da CEV mantevese, inicialmente, inarredável na decisão de vacinar toda a população, para depois implantar as etapas de vigilância epidemiológica e de consolidação. O principal responsável pela estruturação da CEV e defensor dessa estratégia, Oswaldo José da Silva, baseava-se em sua enorme experiência no controle da malária, inclusive na erradicação do Anopheles gambiae no Nordeste brasileiro. Enquanto isso, a Organização Mundial de Saúde (OMS) defendia a adoção de estratégia testada na África, que demonstrara a eficácia da vacinação focal, restrita aos contatos dos casos detectados e, portanto, orientada pela vigilância epidemiológica ativa. É claro que a organização de campo desenvolvida para o combate a vetores não se aplicava exatamente a um programa de vacinação em massa, no qual a adesão popular seria o fator crítico. Mas a estruturação técnico-administrativa da $\mathrm{CEV}$, originalmente moldada na experiência do combate à malária e à febre amarela, propiciou uma disciplina tática que foi fundamental para o grande êxito obtido e para a formação de profissionais que vieram a se dedicar, posteriormente, ao controle de doenças evitáveis por vacinação.

Que dados orientavam a ação do PNI nos primeiros anos e em que medida o programa contribuiu para aprimorar a vigilância epidemiológica nopaís?

Em 1973, os dados disponíveis, em nível nacional, provinham do Boletim Epidemiológico, criado pela FSESP em 1969 e editado quinzenalmente, que registrava o número de casos de algumas doenças, 
notificados semanalmente pelas secretarias estaduais de Saúde. Nas secretarias estaduais, a consolidação e o envio desses dados cabia às unidades de vigilância epidemiológica (UVE), que foram estimuladas pela CEV com o apoio das diretorias regionais da FSESP, mediante convênio específico firmado entre as duas instituições. Em alguns estados, a FSESP Cedeu seus próprios técnicos para organizar as UVE nas secretarias de Saúde.

Quais as doenças selecionadas para notificação e acompanhamento?

Basicamente as consideradas evitáveis por vacinação: poliomielite, sarampo, difteria, tétano, coqueluche e febre tifóide. Posteriormente, foram agregadas a raiva humana e a doença meningocócica, em função da criação do programa nacional de profilaxia da raiva e da epidemia de meningite. O Boletim consolidava, também, os casos de doenças tratadas por programas federais específicos, como as de transmissão vetorial, a tuberculose, a hanseníase e a varíola. No primeiro grupo de doenças, a notificação não estava inicialmente sujeita a critérios técnicos nacionais, o que dificultava muito as análises comparativas e de tendências. Mesmo a poliomielite, que no período 1971-73 havia sido objeto de um plano nacional que muito contribuiu para aperfeiçoar as estratégias de vacinação, descurou da vigilância epidemiológica. Tampouco havia um sistema de informação nacional que registrasse, sistematicamente, o número de doses de vacinas distribuídas e aplicadas.

Quais as principais dificuldades encontradas na implantação do PNI?

Inicialmente, o PNI enfrentou percalços de ordem institucional, com a mudança do governo federal, em março de 1974. O Ministério da Saúde foi transferido para Brasília e reestruturado. A FSESP, por sua vez, tendo permanecido no Rio de Janeiro, reaglutinou seus profissionais que estavam cedidos a vários órgãos do ministério, como a CEV e a DNEES. Em janeiro de 1973, a FSESP havia recebido delegação formal do Ministério da Saúde para coordenar o PNI e o Sistema Nacional de Vigilância Epidemiológica (SNVE) e, para tanto, adaptou a sua estrutura, transformando o antigo Centro de Investigações Epidemiológicas (CIE) na Divisão de Epidemiologia, Estatística e Informação (DEESI). Embora o governo que assumiu em março tenha ratificado a delegação de competência à Fsesp, o distanciamento geográfico e, também, visões diferentes sobre a condução estratégica do PNI e do SNVE geraram certas dificuldades entre a DeEsi e a então criada Secretaria Nacional de Ações Básicas de Saúde (SNABS), do Ministério da Saúde. Apesar de alguns desencontros, a FSESP conseguiu implementar o Sistema Nacional de Vigilância Epidemiológica, produziu o Guia de Vigilância Epidemiológica, com normas técnicas para todas as doenças, reforçou as UVE estaduais, com apoio técnico-administrativo e atividades de capacitação, organizou o sistema de informação sobre aplicação de 
"No final de

1979, com a epidemia de pólio no Paraná e em Santa Catarina, a proposta de dias nacionais foi aprovada, apesar da oposição de representantes do movimento sanitário, que a consideravam campanhista e contrária à política de descentralização da saúde. $O$ ministério enfrentou 0 debate ... O modelo era tecnicamente correto e não confrontava 0 princípio de gestão descentralizada. $\mathrm{Na}$ realidade, $\mathrm{O}$ fortalecia." vacinas e estruturou os sistemas de apoio laboratorial ao diagnóstico de algumas doenças, como poliomielite e raiva humana. $\mathrm{E}$ ainda produziu análises de situação e fortaleceu as atividades de retroalimentação do SNVE, com a dinamização do Boletim Epidemiológico.

\section{Quer dizer que as mudanças não chegaram a produzir impacto no} PNI?

Houve certo retrocesso nas estratégias de cobertura do PNI. Pretendeu o Ministério da Saúde fortalecer as atividades de vacinação de rotina, na rede de serviços permanentes, em substituição às campanhas que eram realizadas. Para tanto, instituiu instrumentos de regulação, como lei específica que tornou obrigatório o cumprimento do esquema básico de vacinação em menores de um ano de idade, sob pena de suspensão do salário-família. Essa política não se mostrou efetiva, pois a rede de serviços estava despreparada para implementá-la. Em conseqüência, as coberturas vacinais permaneceram insatisfatórias, o que propiciou a ocorrência de várias epidemias, entre elas a de poliomielite.

Como foram criados os dias nacionais? Que pressões ou resistências o ministério enfrentou para realizar a vacinação nacional em um único dia?

A idéia de "dias nacionais de vacinação contra a poliomielite" surgiu em função da experiência anterior com os "dias estaduais", com o Plano Nacional de Controle da Poliomielite. Nos três anos (1971-73) em que essa estratégia foi desenvolvida pelo Ministério da Saúde em cerca de 14 estados, ficou evidente que o modelo tenderia a se esgotar, comprometendo a sua continuidade.

\section{Por quê??}

Por várias razões. Primeiro, o período de duração da iniciativa não estava definido, nem havia uma clara meta a ser alcançada. As datas de vacinação eram estabelecidas por cada estado individualmente, resultando que a campanha ficava sob total responsabilidade das secretarias de Saúde e eventuais mudanças administrativas no nível estadual comprometiam a continuidade do processo. Além disso, a iniciativa era demasiadamente dependente de ajuda voluntária, inclusive da mídia. O plano previa três dias de vacinação por ano, o que aumentava o desgaste da mobilização social. O impacto epidemiológico, por sua vez, era menos evidente, porque a ação não abrangia todo o país, exigindo avaliações em cada estado trabalhado. Não bastasse tudo isso, apenas as áreas urbanas com mais de dois mil habitantes eram incluídas, o que representava outra dificuldade para a avaliação de impacto, e o ministério não estava estruturado para realizar o complexo suprimento de vacinas. 


“No Brasil as
campanhas
produziram efeito
educativo
importante. A
população
incorporou a
vacinação às suas
necessidades de
saúde e passou a
acreditar neste
serviço oferecido
pelo Estado. Os
profissionais da
rede, por sua
vez, tiveram a
auto-estima
valorizada e
adquiriram
experiência com
várias outras
aplicações.”

"No Brasil as campanhas produziram efeito educativo importante. A população incorporou a vacinação às suas saúde e passou a acreditar neste serviço oferecido pelo Estado. Os profissionais da rede, por sua , tiveram vito-estima valorizada e adquiriram experiência com várias outras
Essas evidências reforçavam a tese de uma gestão centralizada em nivel nacional?

Em 1979, a situação da poliomielite no Brasil era nitidamente grave, em função de baixas coberturas vacinais, como já comentado. O panorama nacional da doença estava perfeitamente delineado e divulgado no Boletim Epidemiológico, com base nas ações específicas de vigilância que estavam estabelecidas em todo o país. Epidemias de considerável magnitude ocorreram naquele ano em várias capitais brasileiras, gerando desconforto para os gestores da saúde. De outra parte, algumas premissas operacionais para um programa de grande porte haviam sido cumpridas, como o suprimento regular de vacinas pela CEME e a existência de um poderoso sistema de comunicação social organizado na Presidência da República, que veiculava informações simultaneamente em todo o país. E tínhamos a experiência de outros países latino-americanos, que haviam controlado a poliomielite com campanhas nacionais de vacinação. Em Cuba e na Argentina essa estratégia se mantinha com grande sucesso há anos. No mesmo ano de 1979, um artigo publicado por Albert Sabin no International Journal of Epidemiology fundamentava a proposta e detalhava recomendações sobre a organização de dias nacionais de vacinação, como modelo a ser adotado nos países ainda endêmicos. Na ocasião, o assunto chegou a ser ventilado no Ministério da Saúde do Brasil, sem ser discutido seriamente.

Quando, efetivamente, surgiu a oportunidade de implantar o modelo? Ao final daquele mesmo ano de 1979, quando irrompeu uma epidemia de poliomielite nos estados do Paraná e Santa Catarina. O episódio teve grande repercussão na mídia, no momento em que Waldyr Arcoverde assumia o Ministério da Saúde. A proposta de dias nacionais de vacinação foi discutida e aprovada, embora com a oposição de alguns setores da saúde pública. Representantes do movimento sanitário, que ganhava forte expressão à época, consideravam-na "campanhista" e contrária à política de descentralização da gestão do setor saúde. Segmentos mais conservadores a consideravam ineficaz e antieducativa, tendente a desfocar a atenção sobre os grupos de risco e a afastar a população dos centros de saúde, onde a atenção integral à saúde deveria ser prestada. A direção do ministério enfrentou esse debate, argumentando que se tratava de um modelo tecnicamente correto e que não confrontava o princípio de gestão descentralizada dos serviços, mas, na realidade, o fortalecia.

Como a Organização Mundial de Saúde e a Organização PanAmericana de Saúde receberam esta idéia?

No plano internacional, o Programa Ampliado de Imunização (PAI) fora instituído, na OMS (1974) e na OPAS (1977), segundo os preceitos da atenção primária que vieram a ser consagrados na famosa Conferência 
“Os dias nacionais, instituídos em 1980 , representaram a transição para um modelo operacional que combinava objetividade com regularidade de ação. A repetição sistemática e programada de vacinação em massa substituiu o caráter episódico, tantas vezes criticado, das campanhas tradicionais." de Alma-Ata, em 1978. No entendimento inicial do PAI, métodos considerados verticais, como os utilizados anteriormente na erradicação mundial da varíola (1967-79), deveriam ser substituídos por atividades permanentes na rede básica de saúde, privilegiando a vacinação de rotina e a gestão local de recursos, com controle social. Dessa forma, a receptividade internacional ao modelo de "dias nacionais" foi, inicialmente, reservada. Após a evidência do êxito no Brasil, a OPAS passou a apoiálo, no que foi acompanhada pelo Unicef e, mais tarde, pela OMS.

Em 1992, a OPAS estabeleceu um comitê, presidido por Carlos Taylor, para avaliar a repercussão do Programa Ampliado de Imunização e da campanha de erradicação da poliomielite nos sistemas de saúde do continente americano. A avaliação apontou a mobilização social como um dos principais efeitos positivos da campanha. Qual sua opinião sobre este relatório final do comitê?

Ao propiciar informação objetiva sobre a contribuição do programa de imunização para o desenvolvimento dos sistemas de saúde, o relatório da Comissão Taylor ofereceu subsídio relevante para a harmonização de esforços realizados no continente americano para o efetivo enfrentamento dos problemas de saúde. As conclusões do relatório elucidam alguns aspectos do falso dilema que persistiu durante algum tempo, entre o direcionamento de ações de controle e erradicação de doenças, a implementação dos princípios de atenção primária e o desenvolvimento integral do sistema de saúde. Não há dúvida de que essas abordagens são complementares e sinérgicas, se conduzidas articuladamente. No Brasil, a prática mostrou que as campanhas de vacinação produziram efeito educativo importante, ao contrário do que afirmavam alguns opositores da medida. A população incorporou a vacinação às suas necessidades de saúde e passou a acreditar nesse serviço oferecido pelo Estado. Os profissionais da rede de serviços, por sua vez, tiveram a sua auto-estima valorizada e adquiriram uma experiência com várias outras aplicações.

Que atualizações o senhor proporia ao relatório, após 11 anos de sua publicação?

Nos 11 anos decorridos desde a investigação Taylor, o sistema de saúde brasileiro passou por profundas transformações. A implementação do SUS introduziu novos elementos na prestação de serviços de imunização, como o fortalecimento da gestão municipal, o financiamento da atenção básica e a pactuação de ações nas esferas nacional e estadual, em instâncias permanentes. Essa experiência fez amadurecer o entendimento sobre processos comuns de trabalho, enquanto alguns objetivos e estratégias nacionais, como o PNI e os dias nacionais de vacinação, continuam vigentes. Assim sendo, a realização de um novo estudo, nos moldes da Comissão Taylor, certamente traria à discussão outras contribuições importantes. 
A análise retrospectiva do PNI tem suscitado alguns questionamentos sobre os efeitos da Campanha de Erradicação da Poliomielite e de outras campanhas (febre amarela, meningite ou rubéola, por exemplo). Para alguns, a lógica das campanhas impede o PNI de se enraizar na estrutura dos serviços de saúde. Para outros, o problema está no fato de o PNI ainda não tirar o devido proveito de ações mais focais. Como o senhor vê essa questão?

Essa questão acompanha a trajetória do PNI desde as suas origens. Como conciliar o cumprimento dos objetivos do PNI com a plena inserção das atividades nos serviços permanentes de saúde? Minha percepção pessoal é de que o processo desenvolvido, com êxito, na área de imunização, seguiu no Brasil um curso próprio, diferenciado de outras ações de atenção básica à saúde. Isso se deveu a características muito peculiares da área de imunização, e ao desenvolvimento de um modelo operacional capaz de superar desigualdades regionais muito acentuadas, fazendo-se uso apropriado do forte poder indutor do governo federal. Apesar dos avanços recentes na descentralização do sistema nacional de saúde, há um longo caminho a percorrer até que os sistemas locais de saúde assumam integralmente, e de forma homogênea, a plenitude das ações do programa. Por muito tempo ainda, deverão coexistir as duas abordagens que, entretanto, são conciliáveis e podem potencializar-se mutuamente.

Por que a ação local não é eficaz?

A plena efetivação dos objetivos do PNI significa, em primeiro lugar, o controle ou a erradicação de doenças evitáveis por imunização. Essa é a razão de ser do uso sistemático de vacinas no sistema público de saúde, que implica o máximo aproveitamento de um recurso tecnológico ímpar, em termos de custo-efetividade, mas sujeito à administração rigorosa. Importa também reconhecer que o objetivo de controlar essas doenças transcende a atuação dos serviços locais, por requerer enfoques diferenciados segundo a epidemiologia de cada doença, as propriedades de cada vacina, a capacidade operativa dos serviços em cada nível e o monitoramento atento da situação. A longa experiência do PNI mostra, por exemplo, que na erradicação da varíola a vacinação sistemática teve papel subsidiário, diante da maior eficácia de medidas circunscritas, orientadas pela busca ativa de casos suspeitos. Situação diversa da erradicação da poliomielite, que dependeu decisivamente de melhor aproveitamento das propriedades da vacina oral de vírus vivos, como sua fácil administração e capacidade de propagação ambiental. Já a eliminação do sarampo demandou a remodelagem dos componentes de vigilância, diagnóstico laboratorial, imunização em massa e em rotina, em função de características peculiares da doença. 


“O grande
desafio é fazer o
melhor uso
possível dos
recursos e da
tecnologia, sem
perder de vista o
ideário da
atenção integral,
universal e
descentralizada.
As campanhas
nacionais e o
fortalecimento de
serviços
permanentes não
são estratégias
antagônicas.”

"O grande melhor uso

possível dos

recursos e da

tecnologia, sem

ideário da

atenção integral,

universal e

descentralizada.

As campanhas

nacionais e o

fortalecimento de

serviços

são estratégias

antagônicas."
Estes enfoques diferenciados exigem gestão centralizada?

A condução estratégica das ações em todo o país e o gerenciamento integrado de esforços, em todos os níveis, são aspectos essenciais para assegurar padronização técnico-normativa, suprimento e controle de qualidade dos produtos imunobiológicos, mobilização de esforços intra e intersetoriais, vigilância epidemiológica, avaliação e controle de resultados. Devem estar previstas, ainda, ações supletivas para sanar dificuldades locais, uma vez que desacertos em determinadas áreas podem comprometer a eficiência alcançada em outras áreas, contíguas ou não. Trata-se, enfim, da capacidade de direcionar esforços, em tempo oportuno, para microáreas e grupos populacionais mais vulneráveis. No Brasil, o papel da coordenação central é crítico, pois as condições geográficas, demográficas, socioeconômicas, políticas e organizacionais do país conformaram um modelo de saúde caracterizado, historicamente, pela descontinuidade de iniciativas, fragilidade da rede de serviços permanentes e heterogeneidade de seu funcionamento. Esses fatores condicionaram, a partir da década de 1930, a organização de programas federais autônomos para controlar doenças específicas. No caso da imunização, as experiências pioneiras antes mencionadas abriram caminho para o desenvolvimento de um modelo que procurava conciliar intervenções específicas com atividades integradas.

\section{Em que os dias nacionais se diferenciam das antigas campanhas tradicionais?}

Os "dias nacionais de vacinação contra a poliomielite", instituídos em 1980, representaram a transição para um modelo operacional que combinava a objetividade com a regularidade das ações. A repetição sistemática e programada de operações de vacinação em massa substituiu o caráter episódico, tantas vezes criticado, das campanhas tradicionais. O êxito continuado dessa iniciativa inspirou o seu aproveitamento para a aplicação de outras vacinas, e até mesmo para outras ações de saúde pública. Os "dias nacionais de vacinação" passaram a constituir um eixo integrador do PNI, suprindo as deficiências da rotina ao propiciar, por ocasião do atendimento maciço da clientela aos postos, a complementação do esquema básico de vacinação.

\section{Mas essa estratégia responde plenamente aos objetivos do PNI?}

Claro está que ela é incapaz de solucionar, em definitivo, a operacionalização do PNI. Ela não atende à necessidade de aplicação de vacinas em grupos populacionais que não são alvos preferenciais das campanhas nacionais, nem poderia substituir a iniciativa local, no caso de vacinações focais ou de vacinas com indicações específicas. Além disso, o controle de algumas doenças-alvo do PNI não pode prescindir de ações assistenciais, como a atenção pré-natal para prevenir o tétano do recém-nascido. Outros componentes do controle 
das doenças evitáveis por vacinação, como a vigilância epidemiológica, também dependem essencialmente da iniciativa local. Enfim, nenhuma estratégia intervencionista pode prescindir da eficiente gestão local de serviços, prioridade inadiável do SUS.

Então, o que o senhor postula é a convivência de dois modelos que, em verdade, podem se complementar?

O grande desafio é fazer o melhor uso possível dos recursos e da tecnologia disponíveis, sem perder de vista o ideário da atenção integral, universal e descentralizada à saúde. As campanhas nacionais de vacinação e o fortalecimento de serviços permanentes não são estratégias antagônicas em essência, pois a busca de objetivos nacionais é legítima e inerente ao sistema nacional de saúde, em todos os níveis, e a mobilização nacional, sob gestão descentralizada, fortalece o poder de articulação do setor saúde no nível local. De outra parte, o PNI atingiu um grau de reconhecimento junto à sociedade brasileira, em termos de oferta universal de vacinas e de controle de doenças, que não pode admitir retrocessos. A questão é como aperfeiçoar a capacidade de gestão do programa em todos os níveis do sistema, de forma a que cada qual possa melhor cumprir suas funções próprias. Como os recursos destinados à saúde serão sempre insuficientes para atender às necessidades crescentes do setor, é imprescindível que os esforços sejam bem articulados e que as ações tenham continuidade. As experiências exitosas no campo da saúde pública devem ser sempre valorizadas, pois elas só ocorrem como resultado do idealismo e de muito esforço coletivo.

\section{A que o senhor atribui a descontinuidade recorrente das ações de saúde} no Brasil?

É necessário evitar que a defesa intransigente de certos conceitos inteiramente válidos, mas não plenamente aplicáveis à realidade do país em dado momento - venha a comprometer futuramente o cumprimento das metas do PNI. Exemplos negativos os temos, na saúde pública brasileira, frutos do descompromisso com a história e com a objetividade que deve pautar a gestão pública. O esforço que vem sendo realizado no Brasil para desenvolver o Sistema Único de Saúde (SUS), desde 1990, certamente poderia ter-se beneficiado de conhecimentos adquiridos em iniciativas que, mesmo consolidadas, foram desmerecidas por razões alheias ao seu mérito intrínseco. Por exemplo, a experiência inigualável da Fundação SESP, durante quase cinqüenta anos, na organização e gestão descentralizada de serviços integrais de saúde e saneamento, certamente teria concorrido para superar muitos problemas atuais na operação do SUS. Da mesma forma, o programa de controle da tuberculose, pioneiro em metodologias de programação, implementação, supervisão e avaliação de atividades descentralizadas de controle de doenças, na rede geral de serviços. 
Para atender ao PNI, o Brasil investe mais recursos na compra de vacinas do que no desenvolvimento de pesquisas e na capacitação tecnológica da produção de imunobiológicos. Quais as implicações da dependência tecnológica no desempenho do PNI e que medidas poderiam ser tomadas para reduzir o atual grau de dependência?

Desde a década de 1980, quando o PNI se conformou como um programa de atendimento universal às necessidades da população, ficou caracterizada a importância estratégica de um programa nacional de produção, controle de qualidade e desenvolvimento tecnológico dos produtos imunobiológicos utilizados no país. E isso por várias razões. Primeiro, porque o processo de desenvolvimento científico e tecnológico nessa área gera crescente oferta internacional de novos produtos, com conseqüente pressão de demanda interna, a ser respondida pelo PNI. Segundo, porque a escala das necessidades brasileiras é tão grande que não pode depender exclusivamente da disponibilidade internacional, impondo-se certa autonomia de produção interna. Terceiro, porque a tecnologia já incorporada à produção nacional não pode tornar-se obsolescente, devendo acompanhar os avanços mundiais. Quarto, porque o indispensável controle nacional de qualidade dos produtos utilizados no PNI exige domínio atualizado das tecnologias de produção. Quinto, porque as necessidades brasileiras são por vezes peculiares, não correspondendo à oferta internacional de produtos, impondo-se iniciativas internas de desenvolvimento científico e tecnológico.

O Brasil está capacitado a alcançar auto-suficiência e a competir internacionalmente nessa área?

O Brasil dispõe de um considerável parque produtor nacional que, sendo pouco atrativo para o setor privado, foi historicamente desenvolvido no setor público. A partir de 1980, com a grande expansão das atividades do PNI, tornaram-se críticas as dificuldades encontradas para atendimento da demanda interna, em termos quantitativos e qualitativos. O Ministério da Saúde instituiu, então, o Programa Nacional de Auto-Suficiência em Imunobiológicos, baseado em investimentos no parque produtor nacional, segundo um plano geral que buscava o cumprimento de metas nacionais, respeitadas as vocações institucionais dos laboratórios existentes. O pleno desenvolvimento desse programa foi dificultado por limitações gerenciais e pela crise que afetou o setor público brasileiro, sobretudo a partir da década de 1990. No cenário internacional, observa-se que os investimentos em pesquisa e em tecnologia de produção de imunobiológicos tendem a concentrar-se em poucos laboratórios privados. O descompasso entre esse cenário externo e a situação encontrada internamente condicionou os laboratórios nacionais a buscarem parceria com as grandes empresas que dominam o mercado internacional de produtos imunobiológicos. Essa tendência parece ser, no momento, irreversível. 
O que assegura, então, o suprimento das demandas da população brasileira?

Essa área deve ser assumida pelo governo federal como sendo de importância estratégica para o desenvolvimento do país. E deve ser tratada em um plano diretor que propicie máximo aproveitamento das potencialidades existentes, mediante a instituição de mecanismos administrativos capazes de assegurar capacidade competitiva aos laboratórios nacionais e incentivar a produção e o desenvolvimento tecnológico.

O senhor condena a descontinuidade administrativa na área da saúde. Esforços de preservação da memória e a difusão da história não contribuiriam para que o Brasil aprendesse e se valesse dos erros e acertos dopassado?

Tradicionalmente, a tomada de decisões no setor saúde brasileiro não leva em conta a análise dos processos históricos que condicionaram uma determinada situação encontrada. A instabilidade institucional e a descontinuidade administrativa, que têm marcado o setor, se associam à despreocupação com a documentação, a avaliação e a análise de ações e programas. Estes são elementos indispensáveis para a gestão segura das questões de saúde. Novas administrações tendem a pautarse, muitas vezes, por percepções circunstanciais, resultando que a experiência adquirida se acumule apenas no plano pessoal, sem ser apropriada institucionalmente. A trajetória do PNI registra vários desses desacertos, que retardaram o cumprimento de metas atingíveis em menor tempo. Apesar disso, os resultados obtidos são extraordinários frente aos de outras iniciativas, porque as circunstâncias favoreceram o aproveitamento de experiências passadas. Mas ainda há muito a ser feito na reconstituição da memória das imunizações no Brasil, a partir de documentação oficial de órgãos que foram extintos ou transformados, sem o devido cuidado com a preservação de seu acervo. Um exemplo é o da erradicação da varíola, um marco importante dessa trajetória e cuja documentação ainda não foi recuperada.

\section{Ficha técnica}

Entrevistado: João Baptista Risi Júnior

Entrevistador: Carlos Fidelis Ponte

Local e data da entrevista: concedida por e-mail em junho 2003

Edição e apresentação: Cristina Chacel 